\title{
Vivid Spectrum of Two Successfully Treated Cases of Post-Transplant Renal Artery Stenting
}

\author{
Ankur Kamra ${ }^{1}$, Sajeev Chakanalil Govindan ${ }^{1}$, Kader Muneer $^{1}$, Sreelatha Melemadathil ${ }^{2}$ \\ Departments of ${ }^{1}$ Cardiology and ${ }^{2}$ Nephrology, Government Medical College, Kozhikode, Kerala-673008, India.
}

\section{Corresponding Author:}

Sajeev Chakanalil Govindan

Email: sajeev.cg@gmail.com

This is an Open Access article distributed under the terms of the Creative Commons Attribution License (creativecommons.org/ licenses/by/3.0).

Received Accepted

Published

June 29, 2018

October 2, 2018

October 30,2018

\begin{abstract}
Background: As renal transplants are increasing day by day, we often encounter ever more complications associated with it. Transplant renal artery stenosis (TRAS) has been considered as one of the most important and preventable vascular complication of renal transplantation. It may develop earlier or later after renal transplantation and both may develop with different etiology and patho-physiology. Currently, percutaneous trans-luminal angioplasty (PTA) with stenting has been considered as most favorable and successful option for managing TRAS. Case Report: Here in present case series, we represent two such cases of different patients: i) a 34-year-old woman successfully underwent PTA. After four months of renal transplant, she developed $80-90 \%$ stenosis of recipient internal iliac artery, and ii) a 15-year-old boy underwent PTA after 10 days of renal transplant for discrete lesion of donor vessel near anastomotic site. Conclusion: TRAS can manifest with the varied presentations and can be treated successfully with timely interventions.
\end{abstract}

Keywords: Angioplasty, Kidney Transplantation, Pathologic Constriction, Renal Artery Obstruction, Stents.

\section{Introduction}

In India due to increase in awareness among the people regarding organ donation after death and availability of new medical techniques, renal transplantation is evolving with great frequency [1]. With the availability of various effective immunosuppressants, the incidence of allograft rejection has decreased making transplant renal artery stenosis (TRAS) one of the most prevalent serious vascular complications of renal transplantation. The TRAS usually appears at any time post-transplantation, but most probably develops between 2-45 months following renal transplant with a prevalence rate of $1-23 \%$ [2]. It has been considered as the major cause of graft loss and premature death of the host, as it presents with worsening/refractory hypertension, allograft dysfunction in absence of rejection, ureteric obstruction or infection [3]. Percutaneous trans- luminal angioplasty (PTA) with or without stenting is considered as the most widely acceptable and successful treatment option for TRAS [4].

Here, we will discuss two cases of different age, sex, symptoms and time of presentation with successful intervention with one year follow-up of TRAS.

\section{Case Reports}

\section{Case-1}

A 34-year-old female with known case of chronic kidney disease (CKD) for last four years underwent cadaveric renal transplant with cold ischemic period of 11 hours. Donor's kidney was transplanted on right iliac fossa with end to end anastomosis of donor renal artery to recipient internal iliac artery and end to side anastomosis of donor renal vein to recipient external iliac vein. Ureteroneocystostomy 
was done with DJ stent. Post-transplantation the patient did not have significant urine outflow, so she was empirically started on alternative day hemodialysis with intra-venous immunoglobulin. Doppler showed adequate perfusion and biopsy was normal. On $12^{\text {th }}$ day, the patient started having adequate urine output and thus slowly hemodialysis was weaned off and discharged with a diagnosis of delayed graft function. But four months after surgery, the patient presented with acute pulmonary edema with hypertension and was placed on hemodialysis. Magnetic resonance angiogram (MRA) was done which showed lumen narrowing of recipient internal iliac artery at anastomosis site. The renal angiogram demonstrated discrete $80-90 \%$ stenosis of recipient internal iliac artery at anastomosis site [Fig.1]. So, the patient was taken for renal angioplasty, lesion was crossed with coronary wire and pre-dilation was done with 4x10 mm Hiryu balloon (Terumo Europe NV, Belgium) and stented with 6x15 mm Hippocampus renal artery stent (Medtronic, MN, USA) [Fig.2]. Post-transplantation and after one year followup, patient is doing well with normal renal parameters.

\section{Case-2}

A 15-year-old boy with family history of CKD, presented with vomiting and fatigue and was diagnosed with CKD. Patient was posted for renal transplant and started on dialysis. Mother was decided as donor and after doing all work up and clearance, patient was posted for live donor transplant. Donor's kidney was transplanted on right iliac fossa with end to end anastomosis of donor renal artery to recipient internal iliac artery, while end to side anastomosis of donor renal vein to recipient external iliac vein. Ureteroneocystostomy was done with DJ stent. After procedure, the patient was kept under observation at nephrology department, his urine output was normal but on day three nephrologists noticed increase in blood pressure with increase in serum creatinine. The

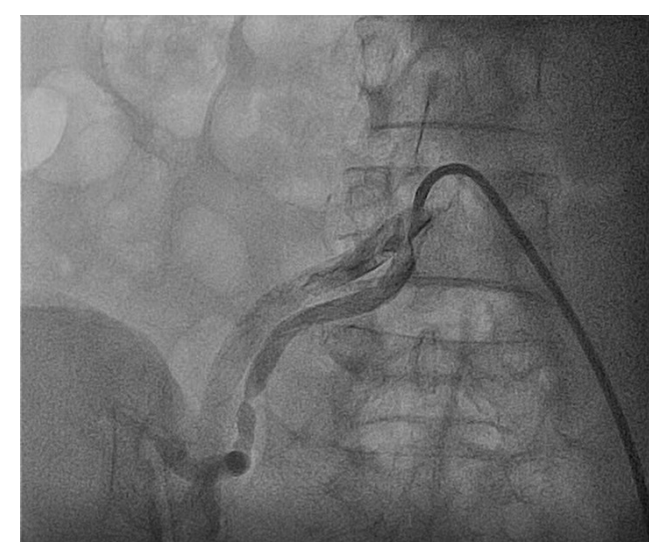

Fig.1: Renal angiogram - showing discrete tight stenosis at anastomotic site.

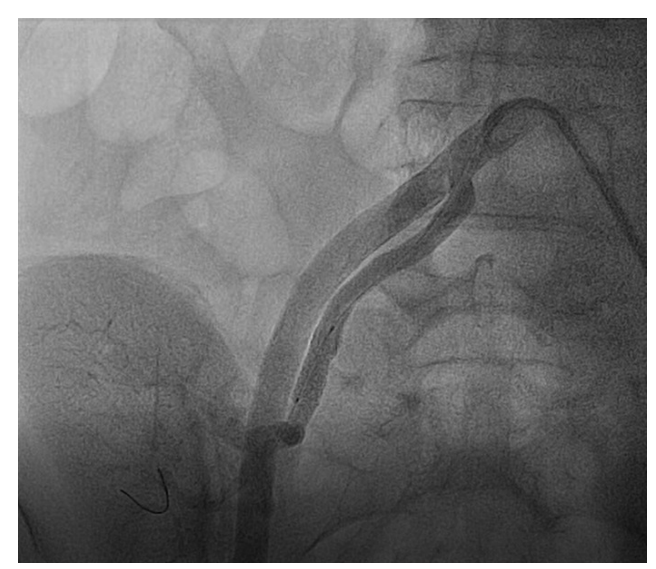

Fig.2: Renal angiogram - showing successful angioplasty done with hippocampus renal artery stent with good distal flow.

MRA showed stenosis at anastomosis of stump of renal artery with donor internal iliac artery. The case was referred to transplant surgeon but they were of the opinion that percutaneous procedure would be better in view of recent surgery. The patient was taken for renal artery stenting on day 11 post-transplant with high risk consent in view of risk of suture and vascular injuries because of very recent surgery. The renal angiogram showed discrete lesion of donor vessel near anastomotic site [Fig.3]. The lesion was crossed with coronary guide wire, predilated with $3 \times 10 \mathrm{~mm}$ noncomplaint balloon at 10 atmospheric pressure and stented with 7x15 mm Herculink Elite renal artery 


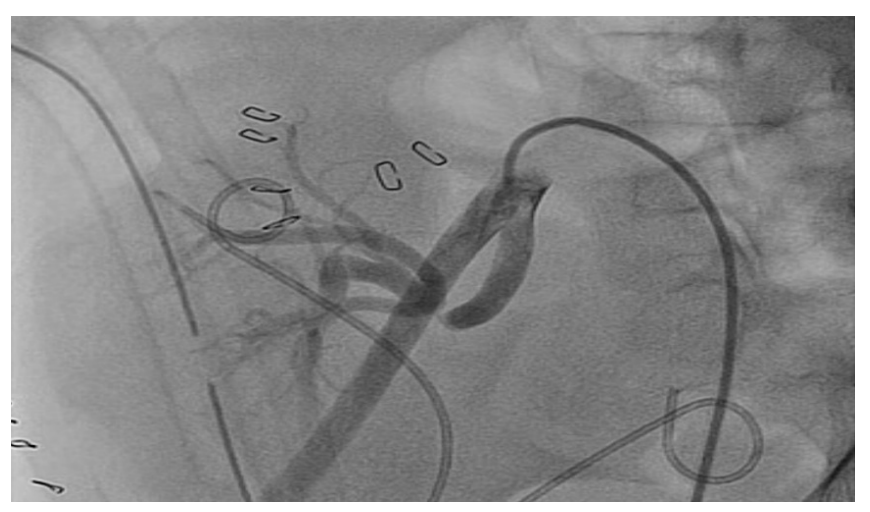

Fig.3: Renal angiogram - showing discrete tight stenosis of grafted vessel just near anastomotic site.

stent (Abbott Vascular, USA) [Fig.4]. After the procedure and after one year follow-up, the patient is stable with normal renal parameters.

\section{Discussion}

The TRAS is the narrowing of the transplant renal arteries which obstruct blood flow to the allograft and ultimately lead to severe hypertension and allograft dysfunction or failure [4]. The stenosis can occur at any place which may appear earlier or later or can be diffused one. The stenosis may be located at the i) pre-anastomotic site which may be due to atherosclerotic disease in the donor vessel, ii) anastomotic site which may be secondary to vessel perfusion injury, faulty suture technique, or reaction to suture material, or iii) post-anastomotic site due to rejection, turbulent flow from kidney malposition, or arterial twisting, kinking, or compression. Late and diffuse stenosis may reflect atherosclerotic disease in the transplant or nontransplant renal artery or the immune mediated endothelial damage $[2,4,5]$. It has been stated in various previous studies that the pervasiveness of TRAS in deceased donor transplants was more prominent compared to living donor transplants which may be due to presence of cold ischemic period in deceased donors $[2,6]$.

Here in the $1^{\text {st }}$ case, a four month's post-renal transplant patient presented with acute pulmonary

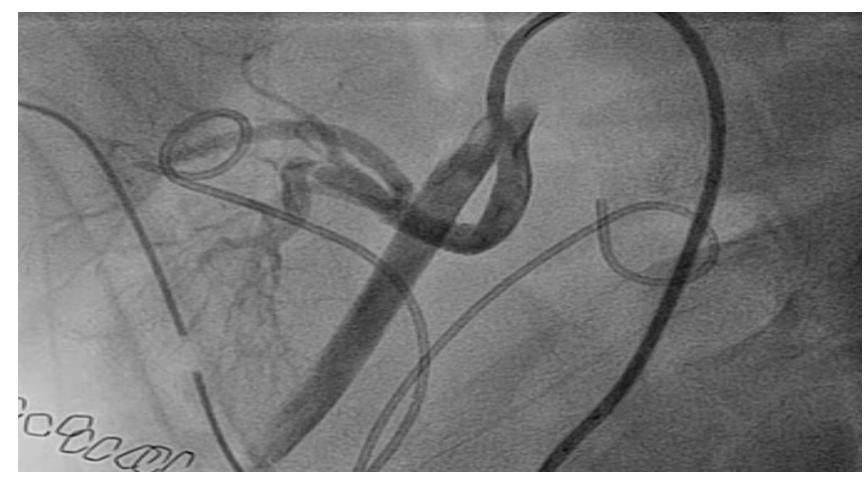

Fig.4: Renal angiogram - showing successful angioplasty done with Herculink elite renal artery stent with good distal flow.

edema with hypertension which were the prime indicators for the presence of TRAS. The reninangiotensin-aldosterone system (RAAS) activation and over-activity of sympathetic nervous system due to stenosis could be the reason for development of hypertension and acute pulmonary edema in the current case. The cause of TRAS in this patient may be the presence of delayed graft function (DGF) owing to the cold ischemia period of 11 hours, as in literature DGF has been considered as the significant risk factor of TRAS [6-8].

The patient in the $2^{\text {nd }}$ case, presented with worsening of blood pressure and higher value of serum creatinine which are considered as very well-known measures of TRAS. In the study by V Audard et al. [7], the clinical presentation of TRAS included refractory hypertension in $75.8 \%$ cases which usually develop within 3-6 months after transplantation, while interestingly in present case the patient developed hypertension within just 10 days of post-renal transplantation. An important cause found in literature for such earlier TRAS was allograft thrombosis which may be caused by technical error, vascular clamp injury, perfusion cannulation injury or vascular abnormalities in the donor kidney [9]. Renal allograft thrombosis may be responsible for upto $35 \%$ of early allograft loss in children which develops with the peak incidence of 48 hours and may be delayed until one week of renal transplantation $[9,10]$. 
The TRAS can be managed by PTA or surgical revascularization along with medical therapy or by medical therapy alone. However, PTA with stent placement is considered as the first line treatment option to correct the stenosis with maximum clinical success rate. The surgical method is the reserved method for unsuccessful or inaccessible angioplasty and very severe cases. In a 10 year retrospective study by Chew et al. [11], the clinical success rate of PTA with stenting was reported to be $76.9 \%$. It has been reported in various series that angioplasty significantly improves the value of blood pressure and also decreases the number of antihypertensive drugs $[8,12]$. Though angioplasty with stenting is generally safe, complications may develop in upto $10 \%$ of cases and $10-33 \%$ incidence of restenosis were also reported $[3,8]$. However, PTA is repeatable and the repeat procedure can provide long-term improvement. In our cases, both patients underwent a successful stent implantation and both are doing well after one year with normal clinical and biochemical parameters.

\section{Conclusion}

TRAS is an important vascular complication of renal transplantation which can manifest with the varied presentations and pathophysiology and can be treated successfully with timely interventions.

Contributors: AK: literature search and manuscript preparation; SCG: literature search, concept and manuscript review and manuscript editing; KM: literature search and manuscript review; SM: patient monitoring and manuscript review. SCG will act as study guarantor. All authors approved the final version of this manuscript.

Funding: None; Competing interests: None stated.

\section{References}

1. Shroff S. Current trends in kidney transplantation in India. Indian J Urol. 2016;32:173-174.

2. Gregor Mlinšek AJ, Damjan Kovač, Jelka Lindič, Miro Mihelič, Nikola Lakič, Aljoša Kandus et al. A complicated case of transplant renal artery stenosis: A case report and literature review. International Journal of Transplantation Research and Medicine. 2015;1:010.

3. Bruno S, Remuzzi G, Ruggenenti P. Transplant renal artery stenosis. J Am Soc Nephrol. 2004;15:134-141.

4. Chen W, Kayler LK, Zand MS, Muttana R, Chernyak $\mathrm{V}$, DeBoccardo GO. Transplant renal artery stenosis: clinical manifestations, diagnosis and therapy. Clin Kidney J. 2014;8:71-78.

5. Akbar SA, Jafri SZH, Amendola MA, Madrazo BL, Salem R, Bis KG. Complications of renal transplantation. Radiographics. 2005;25:1335-1356.

6. Touma J, Costanzo A, Boura B, Alomran F, Combes M. Endovascular management of transplant renal artery stenosis. J Vasc Surg. 2014;59:1058-1065.

7. Audard V, Matignon M, Hemery F, Snanoudj R, Desgranges $\mathrm{P}$, Anglade $\mathrm{M}$, et al. Risk factors and longterm outcome of transplant renal artery stenosis in adult recipients after treatment by percutaneous transluminal angioplasty. Am J Transplant. 2006;6:95-99.

8. Su CH, Lian JD, Chang HR, Wu SW, Chen SC, Tsai $\mathrm{CF}$, et al. Long-term outcomes of patients treated with primary stenting for transplant renal artery stenosis: a 10-year case cohort study. World J Surg. 2012;36:222228.

9. Smith J, Stablein D, Singh A, Harmon W, McDonald R. Decreased risk of renal allograft thrombosis associated with interleukin-2 receptor antagonists: A report of the NAPRTCS. Am J Transplant. 2006;6:585-588.

10. Balachandra $S$, Tejani A. Recurrent vascular thrombosis in an adolescent transplant recipient. J Am Soc Nephrol. 1997;8:1477-1481.

11. Chew LL, Tan BS, Kumar K, Htoo MM, Wong KS, Cheng $\mathrm{C}$, et al. Percutaneous transluminal angioplasty of transplant renal artery stenosis. Ann Acad Med Singapore. 2014;43:39-43.

12. Beecroft JR, Rajan DK, Clark TW, Robinette M, Stavropoulos SW. Transplant renal artery stenosis: outcome after percutaneous intervention. J Vasc Interv Radiol. 2004;15:1407-1413. 